\title{
Determinación de componentes químicos de la madera de cinco especies de encino del estado de Puebla
}

\author{
J. Amador Honorato Salazar ${ }^{1}$ \\ Jorge Hernández Pérez ${ }^{2}$
}

\section{RESUMEN}

Se llevó a cabo un análisis químico para determinar los principales componentes químicos de cinco especies de encinos del estado de Puebla. Las especies estudiadas fueron Quercus affinis, Q. crassifolia, Q. glabrescens, Q. mexicana y $Q$. laurina. Para cada una se determinaron los contenidos de "-celulosa, lignina, pentosanos, extractivos solubles en etanolbenceno, ceniza, sílice y taninos en una mezcla de madera de albura-duramen. También se determinaron los contenidos de extractivos y sílice en madera de albura y duramen, asimismo el contenido de taninos en la corteza fue determinado. Las cantidades de componentes químicos presentes en la mezcla de madera de albura y duramen de las especies se encuentran en el rango de 54.24 a $56.72 \%$ para la " -celulosa, de 19.84 a $22.35 \%$ para la lignina, de 18.37 a $21.64 \%$ para los pentosanos, de 3.84 a $5.24 \%$ para los solubles en etanol-benceno, de 0.87 a $1.38 \%$ para la ceniza, de 0.0040 a $0.0096 \%$ para el sílice, y de 1.17 a $3.46 \%$ para los taninos. Los contenidos de solubles en etanol-benceno y de sílice fueron más altos en la madera de duramen que en la madera de albura de las cinco especies. El contenido de taninos también fue más alto en las cortezas (7.4-10.4\%) que en las mezclas de madera de albura-duramen. El análisis de varianza estadístico de los resultados indicaron que los componentes químicos varían significativamente $(P<$ 0.05 ) entre las especies y el tipo de madera.

\section{PALABRAS CLAVE:}

Encino, Quercus, compuestos químicos, química de la madera, extractivos.

\section{ABSTRACT}

A chemical analysis was carried out to determine the major chemical components of five oak wood species from Puebla, Mexico. The studied oak species were Quercus affinis, $Q$. crassifolia, $Q$. glabrescens, $Q$. mexicana y $Q$. laurina. Contents of "-cellulose, lignin, pentosans, ethanol-benzene extract, ash, silica and tannin were determined for each species in a mixture of sapwood-heartwood. Contents of ethanol-benzene extract and silica were also determined in sapwood and heartwood, as well as bark tannin content. The amount of chemical components found in the mixture of sapwood-heartwood from the five oak wood species were in the range of 54.24 to $56.72 \%$ for alpha-cellulose, 19.84 to $22.35 \%$ for lignin, 18.37 to $21.64 \%$ for pentosans, 3.84 to $5.24 \%$ for

1 Investigador. INIFAP. Campo Experimental San Martinito. Apdo. Postal No. 124. San Martin Texmelucan, Pue. 74000 México.

2 Ex-investigador del Campo Experimental San Martinito.

Manuscrito recibido para su publicación el 14 de Octubre de 1998. 
ethanol-benzene extract, 0.87 to $1.38 \%$ for ash, 0.0040 to $0.0096 \%$ for silica, and 1.17 to $3.46 \%$ for tannin. Heartwood of the five species gave higher values for ethanol-benzene extract and silica content than sapwood. Barks also contained more tannin $(7.4-10.4 \%)$ than the mixtures of sapwood-heartwood. Analysis of variance of the data showed that chemical components did vary among species and type of wood.

\section{KEYWORDS:}

Oak, Quercus, chemical constituents, wood chemistry, extractives.

\section{INTRODUCCION}

En muchos países desarrollados, por ejemplo, Canadá, Gran Bretaña, Francia e Italia, la madera de encinos es utilizada ampliamente en diversos productos de alto valor agregado, como son: chapa para marquetería y cubiertas de contrachapados, tableros enlistonados, muebles, parquet, lambrin, y otros artículos domésticos. En México, a pesar que los encinos están constituidos por más de 150 especies (Martínez, 1981; Nixon, 1993), ocupando una superficie de 24.2 millones de hectáreas, con existencias volumétricas de 298.4 millones de $\mathrm{m}^{3}$ rollo (Díaz, 1983), su madera aún se sigue subutilizando.

La subutilización de esta especie en nuestro país se debe a que: 1) no existe información suficiente para cada una las especies sobre su distribución, abundancia, existencias volumétricas, sistemas de alta productividad, distribución de productos, estadísticas de producción, consumo y mercado, y 2) no existen técnicas y procedimientos tecnológicos adecuados para su procesamiento.

Los encinos, sin embargo, no solamente son fuente de madera $y$ productos maderables, sino también de productos químicos que pueden usarse como materia prima para otros procesos y para la obtención de otros productos, como pueden ser la pulpa y papel, los taninos usados en la industria de la curtiduría, adhesivos, aditivos para la perforación de pozos, etc.

Para conocer que tipo de substancias químicas tienen los encinos, es necesario realizar una caracterización química de las diferentes especies que componen este género, definiendo la composición de la madera en términos de elementos o moléculas identificables. Esto implica el aislamiento, la purificación y la caracterización de los constituyentes químicos de la madera. No obstante, la composición química de la madera no puede ser definida con precisión para un grupo de especies o cierta especie, dado que ésta varía dependiendo de la parte del árbol, tipo de madera, localización geográfica y condiciones de crecimiento. En términos generales, la madera de las especies de clima templado y frío está constituida, en promedio, del 97 al 99\% por compuestos poliméricos o principales que forman la pared celular, entre $0.5 \%$ y $6.6 \%$ por extractivos y entre $0.2 \%$ y $0.5 \%$ por material inorgánico (cenizas, principalmente) (Fengel y Wegener, 1984).

En el caso específico de encinos, estudios realizados en Estados Unidos, Inglaterra y Japón, indican que los compuestos poliméricos varían del 80 al $94 \%$, de los cuales la " -celulosa está entre $31 \%$ y $50 \%$, los pentosanos entre $17 \%$ y $24 \%$, y la lignina entre $19 \%$ y $30 \%$. El contenido de extractivos varía del 1 al $11 \%$ y el material inorgánico del 0.3 al $1.4 \%$ (Fengel y Wegener, 1984; Pettersen, 1984).

En México sólo se han estudiado completamente cuatro especies de encinos, Quercus candicans, $Q$. laurina, $Q$. obtusata y $Q$. resinosa. La cantidad de compuestos principales de la madera de estas varia del $53.9 \%$ al $56.3 \%$ para " -celulosa, del $18 \%$ al 
$21.8 \%$ para pentosanos, y del $15.3 \%$ al $22.6 \%$ para lignina; mientras que los extractivos están entre 7.2 y $9.5 \%$, y el material inorgánico es del 0.5 al $1.17 \%$ (Villalvazo y Faix, 1981; Villalvazo et al., 1981). Estudios adicionales de estas especies también han mostrado que se pueden obtener, pulpas con propiedades físicas aceptables para la producción del papel debido a su buena cohesión al sulfato y a la sosa (Villalvazo et al., 1981). Así mismo, es posible obtener ligninas con mejores características de reactividad que aquellas obtenidas de algunas especies latifoliadas como el haya (Fagus sylvatica) y el abedul (Betula spp.) (Villalvazo y Faix, 1981).

Cuando se toma en cuenta la procedencia de la muestra y el tipo de material, ya sea madera o corteza, la cantidad de compuestos químicos puede variar. Por ejemplo, en las cuatro especies antes mencionadas, la cantidad de extractivos, material inorgánico y taninos es mucho mayor en la corteza que en la madera (Sandoval, 1979), aunque el contenido de los compuestos químicos puede ser algunas veces un poco mayor en la ramas que en la madera del tronco principal a $1.30 \mathrm{~m}$ de altura.

En cuanto al $\mathrm{pH}$, el valor promedio de la madera varia de 2.5 al $6.7 \%$, aunque el rango más aceptado es entre 3.0 y $5.5 \%$ (Stamm, 1964). De acuerdo con Torelli y Nufar (1995b), el valor del pH para la madera de duramen de Quercus anglohondurensis y Quercus skinneri es de 3.9 y 4.3 respectivamente, aunque este valor depende del contenido de extractivos presentes. Torelli y Ñufar (1995a), también reportan que el contenido de cenizas respectivo para cada una de las especies es de $0.31 \%$ y $0.45 \%$, mientras que el contenido de sílice es de $0.003 \%$ y $0.002 \%$.

Dada la escasa información que se tiene de la composición química de los encinos mexicanos, estudios químicos adicionales y complementarios sobre las especies de encinos son necesarios para ampliar el conocimiento de los constituyentes químicos que componen su madera, de tal forma que permitan darle un mayor y mejor uso tecnológico e industrial.

\section{OBJETIVO}

El presente estudio se llevó a cabo para determinar los compuestos químicos de la madera de cinco especies de encinos: Quercus affinis, $Q$. crassifolia, $Q$. glabrescens, $Q$. mexicana y $Q$. laurina, las cuales vegetan en el estado de Puebla. Con ello se pretende de contribuir al conocimiento desde el punto de vista químico, de los encinos del país.

\section{METODOLOGIA}

Para cada una de las especies de encino se determinó el contenido de: cenizas, sílice, substancias solubles en etanol-benceno, taninos, celulosa, pentosanos y lignina. El contenido de sílice y substancias solubles se realizó en albura, duramen y mezcla de albura-duramen. El resto de las determinaciones se realizaron en la mezcla de albura-duramen. La determinación de taninos también se realizó en la corteza.

El sistema de muestreo, la preparación de las muestras y la determinación de la mayor parte de los componentes químicos se llevó a cabo siguiendo las normas TAPPI (Technical Association of the Pulp and Paper Industry, 1978).

Las diferentes normas especifican que cada una de las determinaciones deben llevarse a cabo por lo menos por duplicado. En este estudio, las determinaciones se realizaron por triplicado. Esto permitió hacer análisis de varianza al $5 \%$ de probabilidad entre las diferentes especies para cada uno 
de los compuestos químicos determinados y entre los componentes de las maderas de albura, duramen y mezcla de alburaduramen. También se realizaron comparaciones entre la cantidad de taninos de la madera y la corteza. Para la comparación múltiple de medias se utilizó el método de diferencia mínima significativa (Infante y Zarate, 1997).

Preparación de las muestras para análisis químicos. Las muestras de cada especie se obtuvieron de la primera troza cortada a $30 \mathrm{~cm}$ del suelo y después seccionada a $2.60 \mathrm{~m}$. De acuerdo con la norma TAPPI T 257-os 76, se deben cortar 3 rodajas de $1 / 4$ de pulgada. En este estudio las rodajas se cortaron de 2 pulgadas debido a que los diámetros de las trozas fueron menores a los que especifica la norma, además de considerarse material para imprevistos. Las rodajas se cortaron de la siguiente forma: una del centro y las otras dos aproximadamente a $15 \mathrm{~cm}$ de cada cabezal. Posteriormente, las rodajas se descortezaron y se marcaron cuatro cuadrantes, delineando los cortes por medio de dos líneas que se interceptaban en la médula. Se obtuvieron dos cuadrantes diametralmente opuestos, separándose la albura del duramen y dejándose los otros dos cuadrantes para trabajar con mezcla de albura-duramen. La madera de los cuadrantes se astilló, secándose después al aire libre. Una vez que las astillas estaban secas, se molieron en un molino tipo Wiley y se pasaron a través de una malla No. 40 (0.42 mm).

De acuerdo con la norma TAPPI T 12 os-75, la madera para la mayor parte de los análisis químicos debe estar libre de extractivos; por lo cual la madera molida se sometió a un proceso de extracción por 4 horas con una mezcla recién preparada de etanol-benceno (1:2 v/v), seguido de un lavado con etanol aplicando vacío y de otra extracción con etanol al 95\% durante 4 horas o hasta que la solución resultó incolora. Después se lavó 5 veces con agua destilada por períodos de una hora y se dejó secar al aire libre.

Siguiendo la misma norma, se determinó el contenido de humedad de la madera libre de extractos. Tal determinación consistió en pesar 2 gramos de muestra en un crisol de peso conocido. Posteriormente la muestra se secó en un horno a $105 \pm 3{ }^{\circ} \mathrm{C}$ durante 2 horas, se dejó enfriar en un desecador y se volvió a pesar. Se continuó secando en el horno por períodos de 1 hora hasta que se obtuvo un peso constante.

\section{Determinación de los componentes químicos de la madera.}

1. Determinación de cenizas: Para la determinación de cenizas se usó la norma TAPPI T 15 os-58. Se tomaron $4 \mathrm{~g}$ de madera libre de extractos, colocándose en un crisol de peso conocido. La madera se secó hasta obtener un peso constante. Después se quemó directamente con una flama hasta que ésta desapareció y se introdujo en una mufla a $575 \pm 25{ }^{\circ} \mathrm{C}$ durante un período de 3 horas o hasta que todo el carbón se quemó, indicado por la desaparición de partículas negras. La muestra se dejó enfriar en un desecador y se pesó. El porcentaje de cenizas se calculó en base al peso anhidro de la muestra.

2. Determinación de sílice: En la determinación de sílice se utilizó el método fotométrico (Mozina, 1977). En este método, $10 \mathrm{mg}$ de cenizas y $2 \mathrm{~g}$ de una mezcla de ácido bórico y carbonato de sodio se fundieron en un crisol de peso conocido. El material fundido se dejó enfriar en un desecador y después se lavó con 40 $\mathrm{ml}$ de ácido clorhídrico, ajustándose posteriormente a un $\mathrm{pH}$ de 2 con carbonato de sodio y ácido clorhídrico. Se tomaron 10 $\mathrm{ml}$ de la solución y se ajustó nuevamente a un $\mathrm{pH}$ de 4 a 4.3. Se le agregaron $0.5 \mathrm{ml}$ de una solución de molibdato de amonio y 
después de 5 minutos se adicionaron $1 \mathrm{ml}$ de solución de ácido tartárico al $5 \%$ y $0.5 \mathrm{ml}$ de una solución reductora de sulfito de sodio $(0.8 \mathrm{~g})$, agua destilada $(20 \mathrm{ml})$ y ácido amino naftol sulfónico $(0.16 \mathrm{~g})$, aforando con agua destilada a $50 \mathrm{ml}$. Se dejó reposar 20 min y se midió su absorbancia a una longitud de onda de $720 \mathrm{~nm}$. Posteriormente se leyó la concentración en una curva estándar.

\section{Determinación de solubles en etanol-} benceno: La determinación de extractivos solubles en etanol-benceno fue de acuerdo con la norma TAPPI 204 os-76. Aproximadamente $4 \mathrm{~g}$ de madera molida anhidra se depositaron en un dedal de extracción. El dedal se colocó en un equipo de extracción tipo Soxhlet con $150 \mathrm{ml}$ de una mezcla de alcohol-benceno $(1: 2 \mathrm{v} / \mathrm{v})$ y se ajustó el calentador de tal forma que la velocidad de evaporación reciclara el solvente 6 veces por hora. Después de 6 horas, el solvente se evaporó a un volumen de 20 a $25 \mathrm{ml}$ y se transfirió el extracto a un crisol de peso conocido, lavándolo con pequeñas cantidades de solvente fresco. Después se secó por 1 hora a $105 \pm 3{ }^{\circ} \mathrm{C}$. Una determinación en blanco se llevo a cabo para conocer el peso del residuo del solvente. El porcentaje de extractivos se calculó por medio de la siguiente expresión:

$$
\operatorname{Extractivos}(\%)=\frac{\left(P_{e}-P_{r s}\right)}{P_{m}}
$$

donde:

$\mathrm{P}_{\mathrm{e}} \quad=$ Peso anhidro del extracto

$\mathrm{P}_{\mathrm{rs}}=$ Peso anhidro del residuo del solvente

$\mathrm{P}_{\mathrm{m}}=$ Peso anhidro de la muestra de madera molida.

4. Determinación de lignina: Se utilizó la norma TAPPI T 222 os-74 para determinar el contenido de lignina de cada una de las especies de encino. A una muestra de $1 \mathrm{~g}$ del material anhidro libre de extractos se le agregaron $15 \mathrm{ml}$ de ácido sulfúrico al $72 \%$, manteniéndose en agitación en baño maría a $20 \pm 1{ }^{\circ} \mathrm{C}$. Después se transfirió a un matraz con agua destilada (500-400 ml), se enjuagó y diluyó con agua hasta alcanzar una concentración de ácido del 3\% y un volumen de $575 \mathrm{ml}$. Posteriormente se hirvió a reflujo por 4 horas para asegurar un volumen constante de agua. La solución se decantó manteniendo el matraz en una posición inclinada hasta que el material estuvo finalmente dividido, se filtró a través de un crisol tipo Gooch de porosidad fina, se lavó con agua caliente y se secó a $105 \pm$ $3{ }^{\circ} \mathrm{C}$ hasta alcanzar un peso constante. El porcentaje de lignina se calculó como:

$\begin{aligned} \% \text { Lignina }=\quad & \begin{array}{l}\text { Peso de lignina } X 100) / \text { Peso } \\ \text { anhidro de la muestra }\end{array}\end{aligned}$

5. Determinación de celulosa: La determinación de celulosa se llevó a cabo de acuerdo con la norma TAPPI T 203 os74. Cinco gramos de madera anhidra libre de extractos se transfirieron a un matraz, agregando $\mathrm{NaOH}$ al $17.5 \%$ a $25 \pm 0.2{ }^{\circ} \mathrm{C}$. Después de $30 \mathrm{~min}$ se añadieron $100 \mathrm{ml}$ de agua destilada a $25^{\circ} \mathrm{C}$, agitándose con una varilla de vidrio y dejándose en baño maría a $25 \pm 0.2{ }^{\circ} \mathrm{C}$ por otros 30 min para que el tiempo total de contacto con el $\mathrm{NaOH}$ fuera de $60 \mathrm{~min}$. Posteriormente se filtró a través de un crisol de porosidad media, desechando los primeros $10-20 \mathrm{ml}$ y colectando $100 \mathrm{ml}$. Se tomaron $25 \mathrm{ml}$ de la solución y $10 \mathrm{ml}$ de dicromo de potasio 0.5 $\mathrm{N}$ y se transfirieron a un matraz de $250 \mathrm{ml}$. Se agregaron $50 \mathrm{ml}$ de ácido sulfúrico concentrado, se dejó calentar por 15 min, para después agregar $50 \mathrm{ml}$ de agua y dejar enfriarse a temperatura ambiente. Se le agregaron de 3 a 4 gotas de indicador de ferroín y se tituló con una solución de sulfato de amonio ferroso $0.1 \mathrm{~N}$ hasta que adquirió un color púrpura. Una titulación en blanco se llevó a cabo con $12.5 \mathrm{ml}$ de $\mathrm{NaOH} 17.5 \%$ y $12.5 \mathrm{ml}$ de agua. El porcentaje de "-celulosa se calculó como: 


$$
\% \alpha-\text { celulosa }=100-\frac{6.85\left(V_{2}-V_{1}\right) \times N \times 21}{V_{3} P_{m}}
$$

donde:

$\mathrm{V}_{1}=$ volumen de la titulación de la muestra

$\mathrm{V}_{2}=$ volumen de la titulación en blanco

$\mathrm{V}_{3}=$ volumen de la solución

$\mathrm{P}_{\mathrm{m}} \quad=$ peso anhidro de la muestra

$\mathrm{N}=$ normalidad exacta del sulfato de amonio ferroso.

6. Determinación de pentosanos: La hemicelulosa de las latifoliadas está constituida principalmente por pentosanos, por lo que se utilizó la norma TAPPI T-223 os-70. Para la determinación de estas substancias, se tomó una muestra de $1.5 \mathrm{~g}$ de madera anhidra libre de extractos y se colocó en un matraz con $20 \mathrm{~g}$ de $\mathrm{NaCl}$ y 100 $\mathrm{ml}$ de $\mathrm{HCl} 3.85 \mathrm{~N}$. La solución se destiló, marcando el nivel de $\mathrm{HCl}$ en el matraz. La velocidad de destilación fue de aproximadamente $2.5 \mathrm{ml}$ por minuto. El destilado se colectó en un matraz volumétrico inmerso en un baño de hielo. El nivel del ácido se mantuvo constante mediante la adición de $\mathrm{HCl}$ fresco. La destilación duró aproximadamente 90 min. La solución destilada se calentó a $20^{\circ} \mathrm{C}$ y se le agregó $\mathrm{HCl}$ hasta completar $250 \mathrm{ml}$, mezclándose completamente. A $5 \mathrm{ml}$ de esta solución se le añadió $25 \mathrm{ml}$ de orcinol y se colocó en baño maría a $25 \pm 1{ }^{\circ} \mathrm{C}$. Después de $60 \mathrm{~min}$, se le agregó etanol hasta completar $50 \mathrm{ml}$, se mezcló y se regresó al baño maría por otros 60 min y posteriormente se midió la absorbencia de la solución a $630 \mathrm{~nm}$. Adicionalmente, 6 porciones de xilosa pura anhidra en el rango de 10 a $100 \mathrm{mg}$ se destilaron de acuerdo con el procedimiento anterior y se midió la absorbancia a $630 \mathrm{~nm}$, lo cual permitió realizar una curva de calibración. El porcentaje de pentosas se calculó por medio de la siguiente expresión:

Pentosas $(\%)$ = lectura del espectrofotómetro / (10 x peso anhidro de la muestra)

7. Determinación de taninos: Para la cuantificación de taninos, se utilizó la metodología de la ALCA (American Leather Chemist's Association, 1970). Muestras de la mezcla albura-duramen y corteza se molieron y se secaron al aire libre, para después pasarse por una malla No. 20 (0.21 $\mathrm{mm}$ ) y secarse en un horno a $105 \pm 3{ }^{\circ} \mathrm{C}$ hasta alcanzar peso constante. Posteriormente se transfirieron al extractor para taninos donde se sometieron a una destilación a reflujo por 7 horas o hasta que se colectó un volumen de 2 litros. El material colectado se dejó reposar por 12 horas. Se colocaron $100 \mathrm{ml}$ de la solución en un crisol de peso conocido, se metieron al horno por 17 horas a $100^{\circ} \mathrm{C}$ y se pesaron después de sacarlos del horno para cuantificar los sólidos totales. A la solución restante se le agregó caolín y se agitó vigorosamente; posteriormente se filtró durante 1 hora, filtrando solamente fracciones de $40 \mathrm{ml}$, las cuales se regresaron a la solución inmediatamente después de que se filtraron. La filtración se llevó a cabo a $24^{\circ} \mathrm{C}$.

Se obtuvieron $100 \mathrm{ml}$ de la solución, evaporándose por 16 horas a $100{ }^{\circ} \mathrm{C}$ y posteriormente se pesó. Cuando el peso excedía de $0.8 \mathrm{~g}$, la solución se disminuyó a $50 \mathrm{ml}$. El resultado se registró como sólidos solubles.

A la solución proveniente del extractor se le agregó polvo de piel cromatado, agitándose inmediatamente durante $10 \mathrm{~min}$. Después se filtró y se le agregó caolín, filtrándose una vez más hasta que la solución quedó clara. Se secó durante 16 horas a $100{ }^{\circ} \mathrm{C}$ y se pesó. Finalmente se determinó el contenido de humedad del 
polvo de piel, secando $20 \mathrm{~g}$ durante 16 horas a $100^{\circ} \mathrm{C}$.

Las fórmulas utilizadas para el cálculo de taninos son:

$\%$ taninos $=\%$ sólidos solubles $-\%$ no taninos

$\%$ no taninos $=((200+g$ de polvo de piel $) / 200$ $\mathrm{g}$ de no taninos $\times 10$ ) $\times 200$ / peso anhidro de la muestra

$\%$ sólidos solubles $=(2 \times$ peso de los sólidos en $100 \mathrm{ml} \times 100$ ) / peso anhidro de la muestra

\section{RESULTADOS}

Los resultados promedio de los componentes químicos para cada una de las especies de encino y algunos análisis de varianza se presentan en los Tablas 1 a la 6. Asimismo, se presentan gráficas con barras de error estándar para cada uno de los componentes químicos (Fig. 1 a 4). En las tablas de resultados, las letras mayúsculas en negritas representan la comparación entre especies (hileras). Cuando también se incluyen letras minúsculas, éstas representan la comparación entre tipo de madera dentro de las especies (columnas). Para ambos casos, los valores con la misma letra indican que no existe diferencia significativa $(P<0.05)$, ya sea entre especies o entre tipo de madera. Por ejemplo, para el caso de extractivos en la madera de albura (Tabla 3), la letra A indica que no existe diferencia significativa entre los valores promedio de las especies de Quercus affinis, $Q$. glabrescens, $Q$. mexicana y $Q$. laurina; similarmente la letra $\mathbf{B}$ indica que las especies de $Q$. affinis, $Q$. crassifolia, $Q$. glabrescens no presentan diferencias significativas en sus valores promedio. Las diferencias significativas se presentan entre los valores promedio de $Q$. crassifolia con los de $Q$. mexicana y $Q$. laurina $(P<0.05)$. Para el caso de $Q$. affinis, la letra a indica que los valores promedio de la madera de albura y la mezcla de albura-duramen no presentan diferencias significativas; mientras que la letra $\mathbf{b}$ indica que el contenido de extractivos en la madera de duramen es significativamente diferente de los otros tipos de madera $(P<0.05)$.

\section{DISCUSION}

El análisis de varianza (ANOVA) para cada uno de los componentes químicos en la mezcla de albura-duramen mostró que el contenido promedio de lignina, pentosanos, cenizas, extractivos y sílice es estadísticamente diferente $(P<0.05)$ entre las especies de encino. En el contenido de lignina, las especies de $Q$. affinis y $Q$. glabrescens presentaron diferencias significativas con respecto a $Q$. mexicana, cuyo contenido fue mayor que el resto de las especies. El contenido de cenizas presentó diferencias significativas entre $Q$. crassifolia y $Q$. laurina. La cantidad de pentosanos fue mayor en $Q$. affinis, $Q$. glabrescens y $Q$. mexicana que en $Q$. crassifolia y $Q$. laurina (Tabla 1 y Fig. 1 ). Para el contenido de extractivos, $Q$. crassifolia presentó el valor más bajo y fue estadísticamente diferente con $Q$. mexicana y $Q$. laurina (Tabla 3 y Fig. 2). El mayor contenido de sílice se presentó en $Q$. affinis que en el resto de las especies (Tabla 5 y Fig. 3).

Las cantidades de componentes químicos presentes en la mezcla de alburaduramen para las especies estudiadas son similares a aquellos reportados por Villalvazo et al. (1981) para Quercus candicans, $Q$. laurina, $Q$. obtusata y $Q$. resinosa. Aunque el contenido de solubles en etanol-benceno y lignina de $Q$. laurina resultaron ser mayores en este estudio. La variación puede deberse al tipo de madera usado para realizar las determinaciones de los compuestos químicos. 
Tabla 1. Contenidos de "-celulosa, lignina, pentosanos y cenizas en mezcla de alburaduramen (\%).

\begin{tabular}{||l|c|l|c|l||}
\hline \hline ESPECIES & " -CELULOSA & LIGNINA & PENTOSANOS & CENIZAS \\
\hline Q. affinis & 56.72 A & $20.15 \mathbf{B}$ & $20.42 \mathbf{B}$ & $1.12 \mathrm{~A}, \mathbf{B}$ \\
\hline Q. crassifolia & $56.15 \mathbf{A}$ & $21.42 \mathbf{A}, \mathbf{B}$ & $18.46 \mathbf{A}$ & $1.38 \mathbf{A}$ \\
\hline Q. glabrescens & $54.24 \mathbf{A}$ & $19.84 \mathbf{B}$ & $21.64 \mathbf{B}$ & 0.91 A, B \\
\hline Q. mexicana & $55.46 \mathbf{A}$ & $22.35 \mathbf{A}$ & $21.32 \mathbf{B}$ & 1.01 A, B \\
\hline Q. laurina & $56.20 \mathbf{A}$ & $21.46 \mathbf{A}, \mathbf{B}$ & $18.37 \mathbf{A}$ & $0.87 \mathbf{B}$ \\
\hline
\end{tabular}

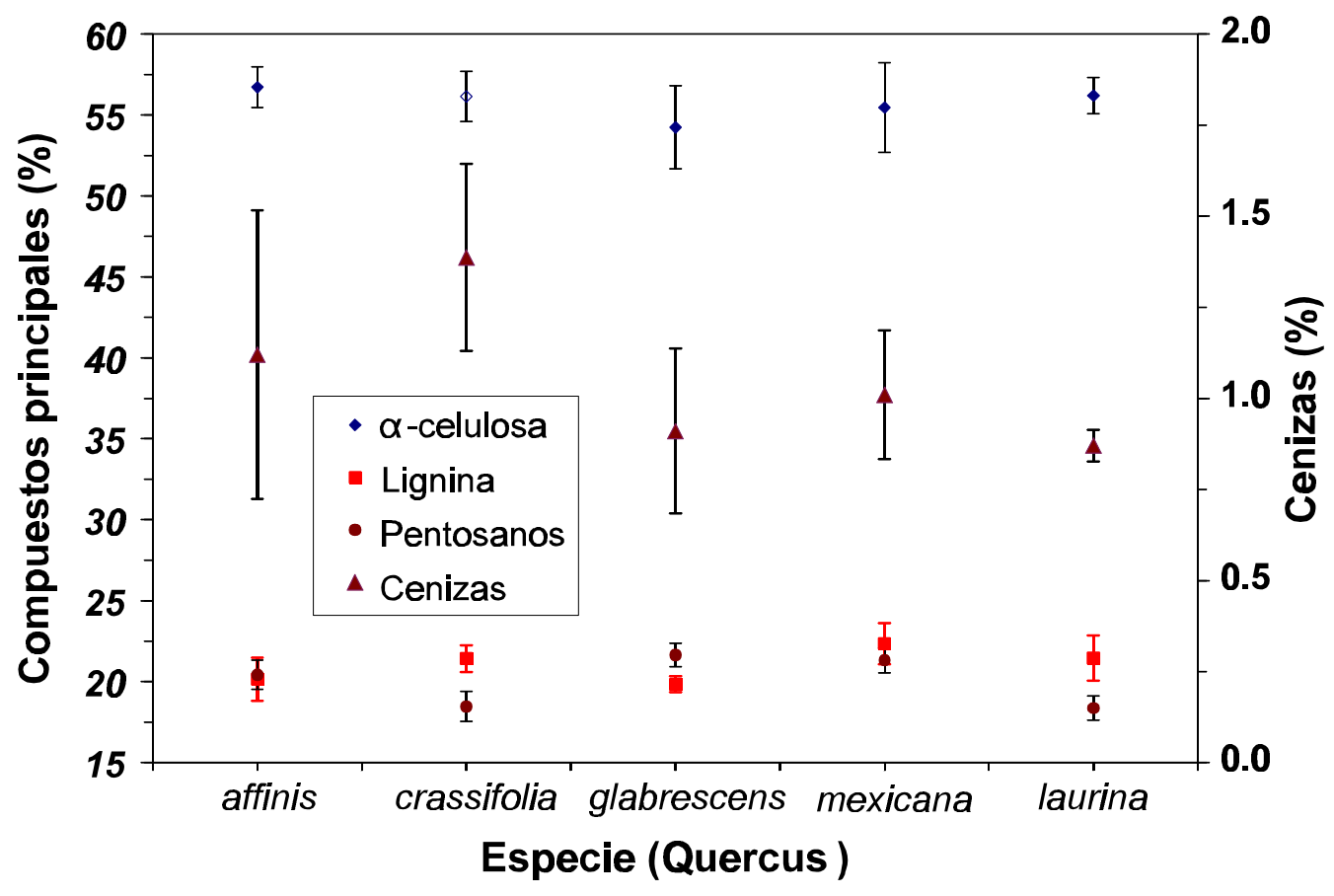

Figura 1. Contenido de compuestos principales y cenizas en la mezcla de albura-duramen. 
Tabla 2. Análisis de varianza para el contenido de extractivos de etanol-benceno en la madera de albura, duramen y mezcla de albura-duramen.

\begin{tabular}{||l|c|r|r|r|r|c||}
\hline \hline $\begin{array}{l}\text { FUENTE DE } \\
\text { VARIACION }\end{array}$ & $\begin{array}{c}\text { GRADOS DE } \\
\text { LIBERTAD }\end{array}$ & $\begin{array}{c}\text { SUMA DE } \\
\text { CUADRADOS }\end{array}$ & $\begin{array}{c}\text { CUADRADO } \\
\text { MEDIO }\end{array}$ & $\begin{array}{c}\text { VALOR } \\
\text { DE F }\end{array}$ & PR > F & $F_{0.05}$ \\
\hline Modelo & 14 & 23.5609 & 1.6829 & 5.33 & 0.0001 & 2.04 \\
\hline Especie & 4 & 16.3713 & 4.0928 & 12.96 & 0.0001 & 2.69 \\
\hline Tipo de madera & 2 & 3.9263 & 1.9631 & 6.22 & 0.0055 & 3.32 \\
\hline Interacción & 8 & 3.2633 & 0.4079 & 1.29 & 0.2850 & 2.27 \\
\hline Error & 30 & 9.4734 & 0.3158 & --- & --- & --- \\
\hline Total corregido & 44 & 33.0343 & --- & --- & --- & --- \\
\hline \hline
\end{tabular}

Tabla 3. Contenido de extractivos en alcohol-benceno por tipo de madera (\%).

\begin{tabular}{||l|l|l|l||}
\hline \multicolumn{1}{|c|}{ ESPECIES } & \multicolumn{1}{|c|}{ ALBURA } & \multicolumn{1}{c|}{ DURAMEN } & \multicolumn{1}{c|}{ MEZCLA } \\
\hline Q. affinis & $4.74 \mathbf{A}, \mathbf{B}, \mathbf{a}$ & $6.02 \mathbf{A}, \mathbf{B}, \mathbf{b}$ & $5.03 \mathbf{A}, \mathbf{a}$ \\
\hline Q. crassifolia & $3.80 \mathbf{B}, \mathbf{a}$ & $3.82 \mathbf{~ D , ~ a ~}$ & $3.84 \mathbf{B}, \mathbf{a}$ \\
\hline Q. glabrescens & $4.15 \mathbf{A}, \mathbf{B}, \mathbf{a}$ & $4.81 \mathbf{C}, \mathbf{D}, \mathbf{b}$ & $4.75 \mathbf{A}, \mathbf{B}, \mathbf{b}$ \\
\hline Q. mexicana & $5.02 \mathbf{A}, \mathbf{a}$ & $6.44 \mathbf{A}, \mathbf{b}$ & $5.24 \mathbf{A}, \mathbf{a}$ \\
\hline Q. laurina & $4.91 \mathbf{A}, \mathbf{a}$ & $5.02 \mathbf{C}, \mathbf{B}, \mathbf{a}$ & $4.68 \mathbf{A}, \mathbf{B}, \mathbf{a}$ \\
\hline
\end{tabular}

Tabla 4. Análisis de varianza para el contenido de sílice en la madera de albura, duramen y mezcla de albura-duramen.

\begin{tabular}{||l|c|r|r|r|r|c||}
\hline $\begin{array}{l}\text { FUENTE DE } \\
\text { VARIACION }\end{array}$ & $\begin{array}{c}\text { GRADOS DE } \\
\text { LIBERTAD }\end{array}$ & $\begin{array}{c}\text { SUMA DE } \\
\text { CUADRADOS }\end{array}$ & $\begin{array}{c}\text { CUADRADO } \\
\text { MEDIO }\end{array}$ & $\begin{array}{c}\text { VALOR } \\
\text { DE F }\end{array}$ & PR $>$ F & F $_{0.05}$ \\
\hline Modelo & 14 & 0.000265 & 0.0000189 & 24.34 & 0.0001 & 2.04 \\
\hline Especie & 4 & 0.000244 & 0.0000611 & 78.54 & 0.0001 & 2.69 \\
\hline Tipo de madera & 2 & 0.000014 & 0.0000073 & 9.34 & 0.0055 & 3.32 \\
\hline Interacción & 8 & 0.000006 & 0.0000008 & 1.06 & 0.4179 & 2.27 \\
\hline Error & 30 & 0.000023 & 0.0000007 & --- & --- & --- \\
\hline Total corregido & 44 & 0.000288 & --- & --- & --- & --- \\
\hline \hline
\end{tabular}




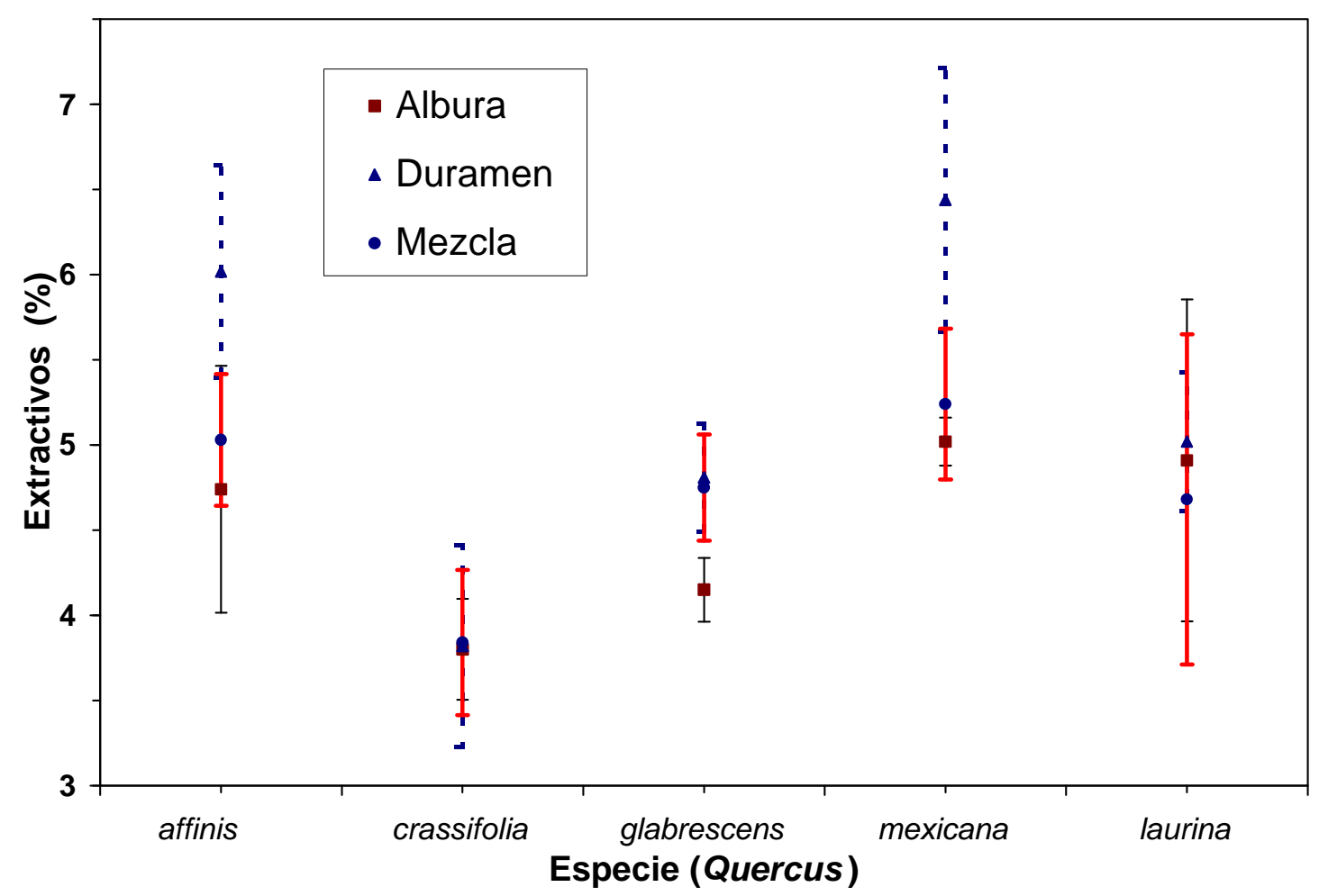

Figura 2. Valores promedio y error estándar del contenido de extractivos en etanol-benceno.

Tabla 5. Contenido de sílice por tipo de madera (\%).

\begin{tabular}{|l|c|c|c||}
\hline \multicolumn{1}{|c|}{ ESPECIES } & ALBURA & DURAMEN & MEZCLA \\
\hline Q. affinis & $0.0083 \mathbf{A}, \mathbf{a}$ & $0.0093 \mathbf{A}, \mathbf{b}$ & $0.0096 \mathbf{A}, \mathbf{b}$ \\
\hline Q. crassifolia & $0.0033 \mathbf{C ,} \mathbf{a}$ & $0.0044 \mathbf{C}, \mathbf{a}$ & $0.0043 \mathbf{C}, \mathbf{a}$ \\
\hline Q. glabrescens & $0.0066 \mathbf{B}, \mathbf{a}$ & $0.0073 \mathbf{B}, \mathbf{a}$ & $0.0070 \mathbf{B}, \mathbf{a}$ \\
\hline Q. mexicana & $0.0010 \mathbf{D}, \mathbf{a}$ & $0.0030 \mathbf{C}, \mathbf{b}$ & $0.0040 \mathbf{C}, \mathbf{b}$ \\
\hline Q. laurina & $0.0037 \mathbf{C}, \mathbf{a}$ & $0.0046 \mathbf{C}, \mathbf{a}$ & $0.0044 \mathbf{C}, \mathbf{a}$ \\
\hline
\end{tabular}


Tabla 6. Contenidos de taninos (\%).

\begin{tabular}{||l|l|l||}
\hline \multicolumn{1}{|c|}{ ESPECIES } & \multicolumn{1}{c|}{ CORTEZA } & \multicolumn{1}{c|}{ MEZCLA } \\
\hline Q. affinis & $10.42 \mathbf{A}, \mathbf{b}$ & $3.46 \mathbf{A}, \mathbf{a}$ \\
\hline Q. crassifolia & $7.40 \mathbf{C}, \mathbf{b}$ & $1.52 \mathbf{B}, \mathbf{C}, \mathbf{a}$ \\
\hline Q. glabrescens & $9.43 \mathbf{A}, \mathbf{B}, \mathbf{b}$ & $2.63 \mathbf{A}, \mathbf{B}, \mathbf{a}$ \\
\hline Q. mexicana & $7.95 \mathbf{B}, \mathbf{C}, \mathbf{b}$ & $2.14 \mathbf{A}, \mathbf{B}, \mathbf{C}, \mathbf{a}$ \\
\hline Q. laurina & $8.62 \mathbf{B}, \mathbf{C}, \mathbf{b}$ & $1.17 \mathbf{C}, \mathbf{a}$ \\
\hline
\end{tabular}

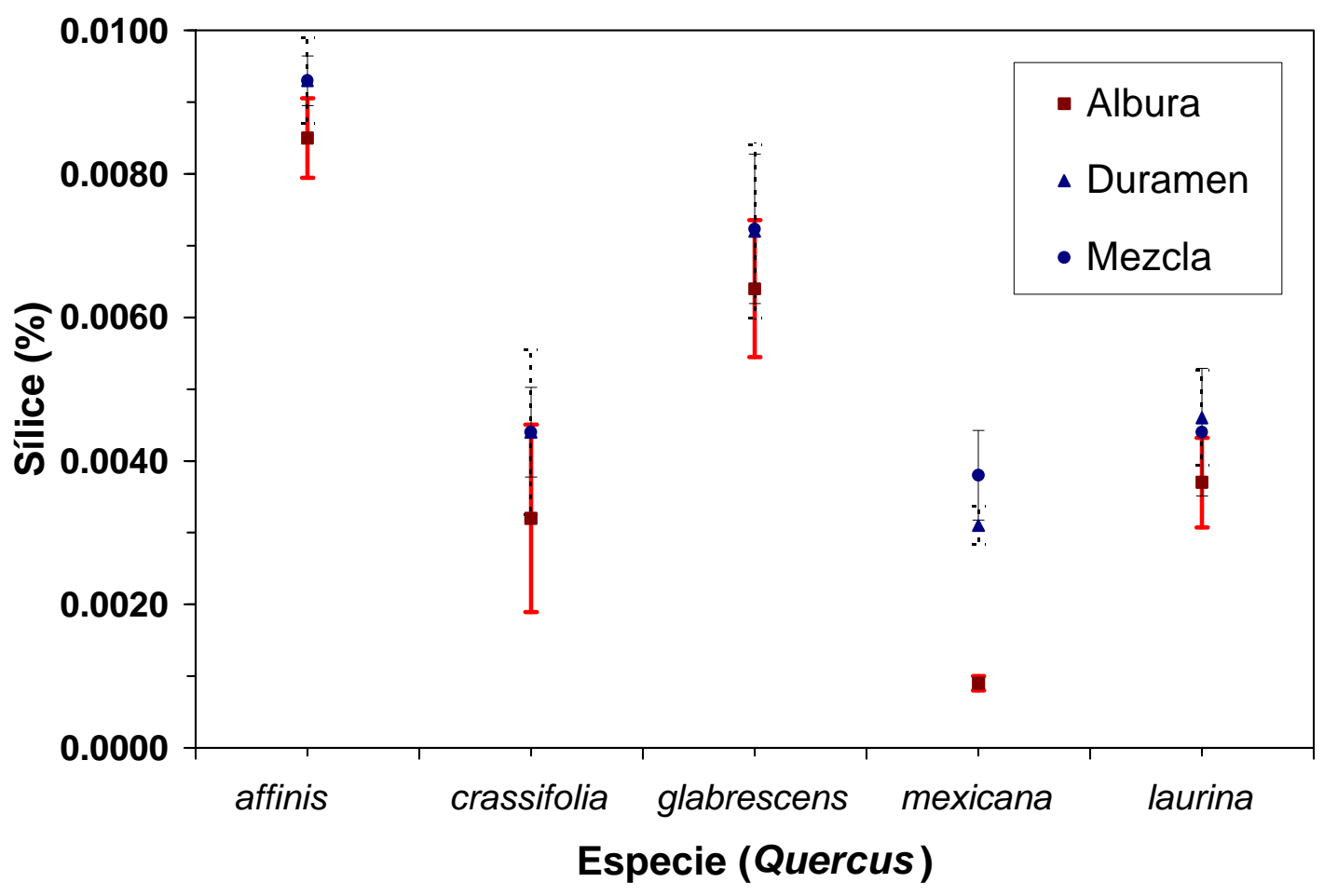

Figura 3. Valores promedio y error estándar del contenido de sílice. 
Una de las características de las especies estudiadas en el presente trabajo, así como las que reportan Villalvazo et al. (1981), es que el contenido de " -celulosa es mayor al que se reporta para especies europeas y estadounidenses. Quizá la localización geográfica y las condiciones de crecimiento de los árboles influya en la cantidad de este componente. Sin embargo, se requieren estudios adicionales en otras especies de encinos mexicanos de diferentes localidades para encontrar un patrón más definido en la composición química de este género.

Los contenidos de " -celulosa de todas las especies estudiadas están arriba del $50 \%$, lo cual puede ser considerable para su utilización en la producción de papel, aunque habría que hacer pruebas de pulpeo y físicas del papel, ya que el encino es de fibra corta y causará efectos no deseados en algunas propiedades físico-mecánicas del papel, pero podría mezclarse con especies de fibra larga para obtener mejores resultados.

Al considerar el contenido de extractivos en alcohol-benceno que se puede obtener de las especies y del tipo de madera (Tabla 2), se observa que existe una mayor variabilidad entre las especies que entre el tipo de madera considerado. La variación entre especies es mayor en la madera de duramen que en la albura o la mezcla (Tabla 3). La diferencia entre el tipo de madera fue solamente para $Q$. affinis, $Q$. glabrescens y $Q$. mexicana, presentándose un mayor contenido de extractivos en la madera de duramen que en los otros dos tipos de madera. Esto se debe a que los compuestos que caracterizan a los extractivos de las especies se encuentran más concentrados por deposición en el duramen, con cantidades menores en la albura (Browning, 1975).

El contenido de sílice también presenta una mayor variación entre especies que entre el tipo de madera (Tabla 4). En este caso la mayor variación se presenta en la madera de albura de las especies. Entre el tipo de madera, la variación sólo se presentó en $Q$. affinis y $Q$. mexicana (Tabla $5)$. La variación en el contenido de sílice puede deberse a las condiciones ambientales y de crecimiento de los árboles, así como a la deposición discreta en las diferentes partes dentro de los árboles (Buchanan, 1975).

Si se considera el contenido de sílice global de las cinco especies, éste varía de 0.0010 a $0.0096 \%$. Estos valores son menores a los que reportan Torelli y Nufar (1995a) para encinos tropicales. Algunos autores consideran que un contenido de sílice entre 1 y $3 \%$ representa problemas para la transformación de la madera, principalmente en el desafilado de las herramientas de corte, pero la mayoría coincide en un porcentaje del $0.5 \%$. Con base en estos valores podría decirse que las cinco especies de encino no presentarían problemas para su procesamiento con las diferentes herramientas de corte. Sin embargo, los valores del contenido de sílice son especulativos, ya que no ha habido estudios cuantitativos directos que correlacionen el contenido de sílice y el desgaste de las herramientas de corte. Esto se debe a que el contenido de sílice puede variar de una parte del árbol a otra, como en el caso de la albura y el duramen de algunas especies. Sin embargo, de manera indirecta Krilov (1980) señala que la rapidez de desafilado de la sierra banda es proporcional al contenido de sílice en la madera.

La variación del contenido de taninos entre especies se presenta tanto en la mezcla de madera como en la corteza (Tabla 6 y Fig. 4). Los valores mostraron ser estadísticamente significativos $(P<0.05)$ para las cinco especies entre los contenidos de taninos de la mezcla de madera y la corteza. Este comportamiento también fue observado por Sandoval (1979) en Quercus candicans, $Q$. laurina, $Q$. obtusata y $Q$. 
resinosa, aunque el contenido de taninos determinado fue mayor que el de este estudio.

De acuerdo con Rowe y Conner (1979), el porcentaje de taninos en la corteza considerados con potencial comercial es de $8 \%$. Esta cifra es relativa debido a las condiciones del mercado, pero si se toma como referencia el presente estudio, las cortezas de las 5 especies podrían considerarse como productoras potenciales de taninos, ya que la mayoría presentan porcentajes mayores o cercanas al $8 \%$. Sin embargo la utilización de la corteza requiere primero de pruebas de pureza de los taninos, estabilidad de la solución, color de la piel curtida y reactividad al formaldehído para su uso en adhesivos.

La variación en los resultados obtenidos es importante porque indica que el estudio de especies de encinos se debe de realizar tanto en albura como en duramen, así como en mezcla de alburaduramen. De esta forma se tendrá un mejor conocimiento sobre la composición química de las especies.

\section{CONCLUSIONES}

La cantidad de componentes químicos presentes en la mezcla de madera de albura y duramen para las cinco especies de encino son en general similares a los que se reportan en la literatura para encinos de México y de otros países. Los contenidos de los principales componentes químicos fluctuaron de 54.24 a $56.72 \%$ para la " celulosa, de 18.37 a $21.64 \%$ para los pentosanos, de 19.84 a $22.35 \%$ para la lignina, de 3.84 a $5.24 . \%$ para los extractivos solubles en etanol-benceno, de 0.87 a $1.38 \%$ para la ceniza, de 0.0040 a $0.0096 \%$ para el sílice, y de 1.17 a $3.46 \%$ para los taninos.
Con excepción del contenido de " celulosa, la cantidad de componentes químicos en los diferentes tipos de madera presentaron una variación estadística significativa $(P<0.05)$ entre especies y dentro de las especies.

Aún cuando no se presentó una diferencia estadística significativa entre los tipos de madera dentro de cada una de las especies, los contenidos de extractivos solubles en etanol-benceno y sílice fueron mayores en el duramen que en la albura. La diferencia significativa en el contenido de extractivos solubles etanol-benceno entre estos dos tipos de madera se presentó en $Q$. affinis, $Q$. glabrescens y $Q$. mexicana, mientras que en el contenido de sílice fue para $Q$. affinis y $Q$. mexicana.

El contenido de taninos fue estadísticamente diferente entre la mezcla de albura-duramen y la corteza para las cinco especies, siendo mayor en la corteza; lo cual concuerda con los resultados obtenidos en otras especies de encinos.

Considerando el contenido de " celulosa en la madera y el contenido de taninos en la corteza que presentan las especies, se podrían utilizar en la producción de papel y taninos. Sin embargo, estudios específicos relacionados con pulpeo y papel, así como de pureza y calidad de los taninos, son necesarios para poder decidir si estas especies son adecuadas o no para la producción de este tipo de productos.

\section{REFERENCIAS}

American Leather Chemist's Association. 1970. Methods of sampling and analysis. Cincinnati, Ohio. 28 p.

Browning, B. L. 1975. The composition and chemical reactions of wood. In: Browning, B. L. (Ed.). The chemistry of 
wood. Robert E. Krieger Publishing Company. New York. p:57-101.

Buchanan, M. A. 1975. Extraneous components of wood. In: Browning, B. L. (Ed.). The chemistry of wood. Robert E. Krieger Publishing Company. New York. p:313-367.

Díaz, G., V. 1983. Informe sobre el aprovechamiento de los encinares de México. CENIPROF, INIF, SFF, $\mathrm{SARH}$. Archivo Técnico. Mimeógrafo. México. 120 p.

Fengel, D. y G. Wegener. 1984. Wood chemistry, ultrastructure, reactions. Walter de Gruyter. Berlin, Alemania. p: 28-65.

Infante, G. S. y G. P. Zárate I. 1997. Métodos estadísticos: un enfoque interdisciplinario. Cuarta reimpresión. Trillas. México, D. F. 643 p.

Krilov, A. 1980. Toward the re-appraisal of the influence of basic wood characteristics on sawblade potential. Holz als Roh-und Werkstoff 38: 145149.

Martínez, M. 1981. Los encinos de México. Anales del Instituto de Biología UNAM. Comisión Forestal del Estado de Michoacán. Serie Técnica Manejo No. 8. 2a Edición. Michoacán, México. 358 p.

Mozina, I. 1977 Research on physical and mechanical properties of tropical woods. Final technical report. University of Ljubijana. $17 \mathrm{p}$.

Nixon, K. C. 1993.The genus Quercus in Mexico. In: Ramamoorthy, T.P. R. Bye, A. Lot y J. Fa (Eds.). Biological diversity of Mexico: origins and distribution. Oxford University Press New York. p: 447-458.
Pettersen, R. C. 1984. The chemical composition of wood. In: Rowell, R. M. (Ed.). The chemistry of solid wood. American Chemical Society. Washington. p: 57-126.

Rowe, W. y A. Conner. 1979. Extractives in eastern hardwoods - a review. General Technical Report FPL 18. USDA-FSFPL-GTR. 67 p.

Sandoval, O. A. 1979. Estudio analítico de substancias extraíbles de cuatro especies del género Quercus. Tesis, Facultad de Química. Universidad de Guadalajara. México. 77 p.

Stamm, A. J. 1964. Wood and cellulose science. The Ronald Press Company. New York. 263 p.

Technical Associaton for the Pulp and Paper Industries (TAPPI). 1978. Preparation of extractive free-wood. TAPPI Test Method T 12 os-75. TAPPI PRESS. Atlanta.

Technical Associaton for the Pulp and Paper Industries. 1978. Ash in wood and pulp. TAPPI Test Method T 15 os58. TAPPI PRESS. Atlanta.

Technical Associaton for the Pulp and Paper Industries. 1978. Alpha-, Betaand Gamma Cellulose in pulp. TAPPI Test Method T 203 os-74. TAPPI Press. Atlanta.

Technical Associaton for the Pulp and Paper Industries. 1978. Alcoholbenzene and dichloromethane solubles in wood and pulp. TAPPI Test Method T 204 os-76. TAPPI Press. Atlanta.

Technical Associaton for the Pulp and Paper Industries. 1978. Acid-insoluble in wood and pulp. TAPPI Test Method T 222 os-74. TAPPI Press. Atlanta. 
Technical Associaton for the Pulp and Paper Industries. 1978. Pentosans in wood and pulp. TAPPI Test Method T 223 os-70. TAPPI Press. Atlanta.

Technical Associaton for the Pulp and Paper Industries. 1978. Sampling and preparing wood for analysis. TAPPI Test Method T 257 os-76. TAPPI Press. Atlanta.

Torelli, N. y K. Nufar. 1995a. Mexican tropical hardwoods. Comparative study of ash and silica content. Holz als Rohund Werkstoff. 53: 61-61.
Torelli, N. y K. Ñufar. 1995b. Mexican tropical hardwoods. pH-value. Holz als Roh-und Werkstoff. 53: 133-134.

Villalvazo, N. J. y O. Faix. 1981. Caracterización analítica de las ligninas de cuatro especies del genero Quercus y sus posibilidades de aprovechamiento. IMCyP Publicación No. 4. Guadalajara, Jal. 7 p.

Villalvazo, N. J., O. Faix, O. y K. A. Grellmann. 1981. Los encinos mexicanos como materia prima para la fabricación de celulosa y papel. IMCyP Suplemento Técnico No. 1.8 p. 
